\title{
Simulation of the performance of Phase-Sensitive OTDR Based on Ultra-weak FBG Array using Double Pulses
}

\author{
Tao Liu, ${ }^{1}$ Feng Wang,,${ }^{1, *}$ Quan Yuan, ${ }^{1}$ Yu Liu, ${ }^{1}$ Lin Zhang, ${ }^{2}$ Xuping Zhang ${ }^{1,3}$ \\ ${ }^{1}$ Institute of Optical Communication Engineering, College of Engineering and Applied Sciences, \\ Nanjing University, Nanjing, 210008, China \\ ${ }^{2}$ Aston Institute of Photonic Technologies, Aston University, Birmingham B4 7ET, U.K \\ ${ }^{3}$ Key Laboratory of Modern Acoustics, Nanjing University, Nanjing 210093, P. R. China \\ *Corresponding author: wangfeng@nju.edu.cn
}

\begin{abstract}
A phase-sensitive optical time domain reflectometry sensing system based on Ultra-weak FBG Array using double pulses is proposed. The principle of this sensing system is elaborated. A series of simulations are carried out to optimize the parameters to increase the sensing distance of this system. The parameters contain reflectivity of $U W F B G$, the interval between adjacent UWFBGS and pulse width. The optimal reflectivity and pulse width corresponding to different grating space are found out to achieve a longer sensing distance.
\end{abstract}

Keywords: Phase-sensitive OTDR, Ultra-weak FBG Array, Signal-to-Noise Ratio

\section{INTRODUCTION}

Vibration sensing is an important application for distributed optical fiber sensors (DOFSs). Among all the kinds of DOFSs, phase-sensitive optical time-domain reflectometry ( $\Phi-O T D R)$ has the abilities of long-distance distributed sensing, high sensitive detection to vibration and so on. As a result, it has attracted much attention and been applied to many fields [1-4].

The signal to noise ratio (SNR) of $\Phi-O T D R$ is limited by the weak Rayleigh backscattering (RBS) in fiber. In order to improve the SNR, the assistance of an Ultra-weak FBG (UWFBG) array is a potential method. UWFBG array is a recently developed technique. A few of its applications have been reported for multi-points and distributed sensing purposes [5-9]. F. Zhu has proposed a distributed $\Phi-\mathrm{OTDR}$ system which can achieve high-precision dynamic strain measurement by sweeping the laser frequency and using unwrapping algorithms. And L. Xia has analyzed the dynamic range of the system by simulations for a fixed UWFBG interval and different number of UWFBGs [9]. However, the method uses a single optical pulse to generate interference between the reflected signals by two adjacent UWFBGs, so the pulse width should be at least twice times longer than the space interval of two adjacent UWFBGs. When the space interval is large, the peak power of the optical pulse is severely limited to avoid the nonlinear effect. Besides, since a large portion of the reflected signals cannot overlap with each other to generate effective interference signal, the long probe pulse will generate more unwanted RBS, which leads to lower SNR.

In this paper, we put forward a $\Phi$-OTDR system in a sensing fiber integrated with an UWFBG array by using double probe pulse. Detailed simulations are made and shown that the proposed system have better performance such as higher SNR, longer sensing distance, et al. And optimal parameters are obtained for the system.

\section{PRINCIPLE}

The principle of the technique is shown in Fig. 1. The sensing fiber is integrated with UWFBG array which has uniform spatial interval. All the UWFBGs have the same central wavelength and the same weak reflectivity. A double-pulse is used as the probe pulse. The space interval between the two pulses equals two times distance between adjacent UWFBGs. As shown in Fig. 1 , because the reflected signal and the probe pulse transmit in opposite directions, the reflected signal of the front pulse from UWFBG2 and the reflected signal of the rear pulse from UWFBG1 overlap and transmit backward together. Because the coherence length of the lightwave is much longer than the interval of the double pulse, the reflected optical signals will interfere with each other. Such a probe pulse can make the optical pulse have a higher peak power to increase the SNR without inducing nonlinear effect in fiber. The interval of double pulse can be adjusted easily to adapt any UWFBG array with uniform interval.

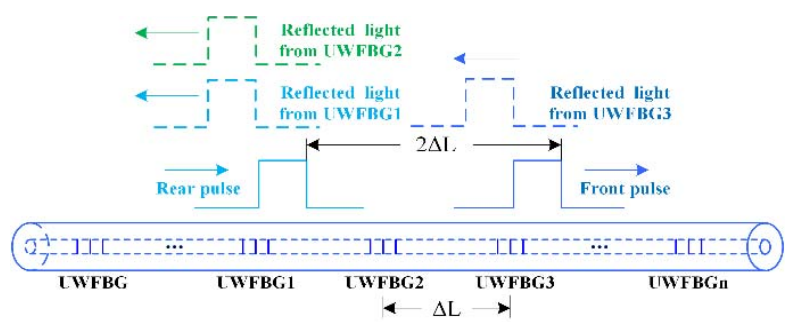

Fig. 1. The principle of the $\Phi-O T D R$ based on UWFBG array using double pulse. 
When the fiber between two adjacent UWFBGs is disturbed by vibration, the refractive index $n$ and the length of the fiber will be changed. Then the phase difference between the two reflected signals will change accordingly, which then induces the variation on the intensity of the interference. So the disturbance of fiber can be detected by detecting the intensity variation of the coherent signal.

\section{SIMULATION}

As is shown in Fig.1, the space interval between adjacent UWFBGs is $\Delta \mathrm{L}$, the number of UWFBGs is $\mathrm{n}$, and thus the distance between the ith UWFBG and the incoming end is $\mathrm{i} \Delta \mathrm{L}$. The reflectivity of all UWFBGs is approximately the same, denoted as $r$, then the transmissivity of UWFBGs is $1-r$. The power of probe pulse is $\mathrm{P}_{0}$, so the reflected signal of the ith UWFBG can be written as

$$
P(i)=P_{R e f}(i)+P_{R a y}(i)+P_{M u l}(i)+P_{N E P}
$$

where $P_{\text {Ref }}(i)$ is the reflected power of the ith UWFBG, $P_{M u l}(i)$ and $P_{\text {Ray }}(i)$ are powers of the multi-reflection lightwave and the RBS respectively, and $P_{N E P}$ is the noise-equivalent-power (NEP) of the detector. So the SNR of the detected signal is defined as

$$
S N R(i)=\frac{P_{R e f}(i-1)+P_{R e f}(i)}{P_{M u l}(i-1)+P_{M u l}(i)+P_{R a y}(i-1)+P_{R a y}(i)+P_{N E P}}
$$

where

$$
\left\{\begin{array}{l}
P_{\text {ref }}(i)=P_{0} r(1-r)^{2(i-1)} e^{-2 \alpha i \Delta L} \\
P_{R a y}(i)=P_{0}(1-r)^{2 i} v_{g} \tau \alpha_{\mathrm{R}} S e^{-2 \alpha i \Delta L} \\
P_{m u l}(i)=P_{0} r^{3}(1-r)^{2(i-2)} \frac{(i-1)(i-2)}{2} e^{-2 \alpha i \Delta L}
\end{array}\right.
$$

and $\alpha=0.046 \mathrm{~km}^{-1}$ is the fiber attenuation coefficient, $v_{g}$ is the velocity of lightwave in fiber, $\tau$ is the pulse width, $\alpha_{R}=3.2 \times 10^{-2} \mathrm{~km}^{-1}$ and $S=1 \times 10^{-3}$ are the Rayleigh scattering coefficient and backscatter capture ratio of fiber respectively $[9,10]$. Based on Eq. (2), simulations were made to investigate the performance of the proposed system. In simulations, the NEP of the detector was assumed to be $-50 \mathrm{dBm}$.

Firstly, the influence of the reflectivity of the UWFBGs is given in Fig.2. It can be seen that a higher reflectivity deplete the power of the probe pulse soon, resulting in a short sensing distance. Meanwhile, a very

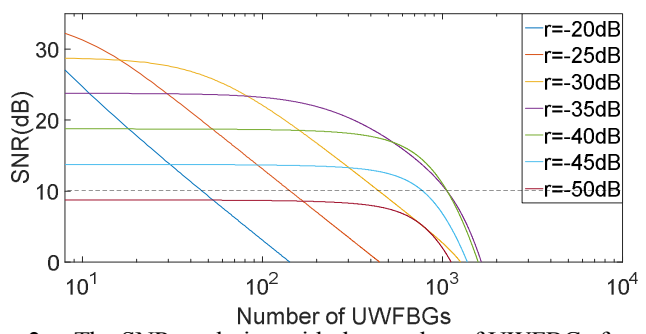

Fig. 2. The SNR evolution with the number of UWFBGs for different reflectivities. The peak power of laser is $20 \mathrm{dBm}$ and the space interval is $50 \mathrm{~m}$. low reflectivity will lead to low SNR and short sensing distance too since the RBS dominates in the signal. Therefore, an optimized reflectivity exists for the longest sensing distance. If we set $10 \mathrm{~dB}$ of SNR as a threshold and make the simulation with fine steps, we find that the optimal reflectivity is $-37 \mathrm{~dB}$ with which the system can reach the longest sensing distance.

Moreover, we find that the optimal reflectivity also changes with the space interval of UWFBGs. Thus, we make simulations to find the relationship between the optimal reflectivity and space interval which is shown in Fig.3. It can be seen that the optimal reflectivity increases with the increase of UWFBG interval. Because when the space interval becomes longer, the loss resulted from UWFBGs' reflection decreases and the power of multi-reflections decreases too for a given length of fiber. Therefore, a stronger reflectivity is allowed to get a longer sensing distance.

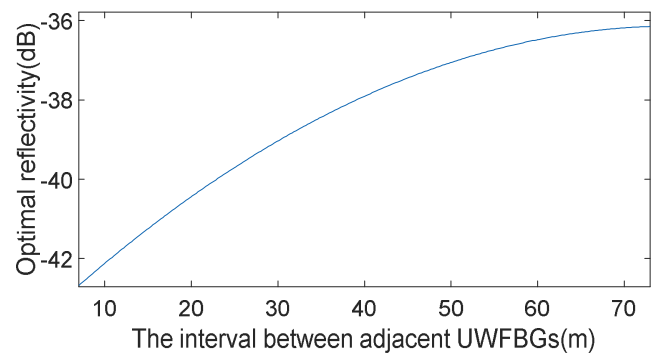

Fig. 3. The relationship between reflectivity and interval. The optimal reflectivity shows the tendency of rising with the increase of interval between adjacent UWFBGs.

Eq. (2) also shows that the pulse width can also influence the SNR. So the following simulations will focus on the influence of pulse width.

Then, the relationship between the pulse width and the SNR of the signal is shown in Fig.4. It can be seen that the SNR decreases with the increment of the pulse width in the front section of fiber. This is because a longer pulse width will generate more RBS. The SNRs for different pulse widths tend to be zero at the same distance, because the power of the probe pulse and the RBS decrease in proportion with the distance while the power of multi-reflections tends to be constant for long distance. From Fig. 4 it seems that the SNR of the



Fig. 4. The SNR evolution with the number of UWFBGs for different pulse widths

proposed technique can be improved by shorten the pulse width and increase the peak power of the probe pulse. However, we can further improve the SNR by average the signals in the same reflected optical pulse obtained with long pulse width. The SNR improvement after digital multiple-point averaging technique is $5 \lg N$ 
[11], where $\mathrm{N}$ is average number. So the SNR improvement with the average for a $100 \mathrm{~ns}$ pulse width is $5 \mathrm{~dB}$ higher than that of a $10 \mathrm{~ns}$ pulse width. Then the relationship in Fig. 4 is amended to Fig. 5 by considering the contribution of average.

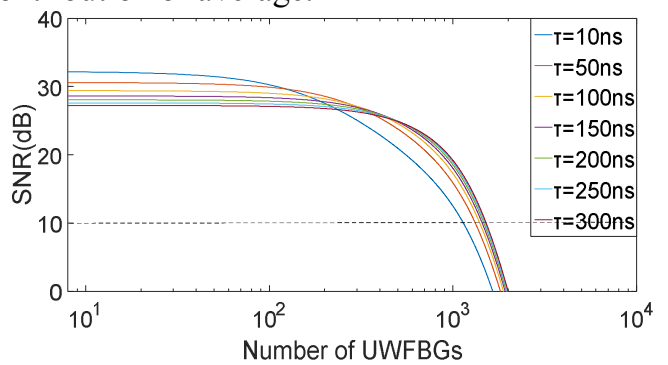

Fig. 5. The SNR evolution with the number of UWFBGs for different pulse widths with average.

During the simulations above, the peak power of the probe pulse is assumed to be constant. As is known to all, the capacity of the maximum peak power in fiber is different for different pulse widths because of nonlinear effect. Normally, the threshold of optical pulse is mainly limited by the stimulated Brillouin scattering and the threshold for different pulse width is shown in Table.1 which is obtained with the parameters in reference [12].

Table. 1 Stimulated Brillouin scattering threshold for different pulse width

\begin{tabular}{cccccccc}
\hline \hline $\begin{array}{c}\text { Pulse } \\
\text { width } \\
(\mathrm{ns})\end{array}$ & 10 & 50 & 100 & 150 & 200 & 250 & 300 \\
\hline $\begin{array}{c}\mathrm{P}_{\text {th }} \\
(\mathrm{dBm})\end{array}$ & 39.5 & 32.5 & 29.4 & 27.7 & 26.5 & 25.5 & 24.7 \\
\hline \hline
\end{tabular}

So in summary, the space interval, the reflectivity, the pulse width and the nonlinear effect are all contribute to the longest sensing distance of the system. And by considering all parameters mentioned above comprehensively, we finally can get the optimal parameters and longest sensing distances for different space intervals, which are shown in Table 2.

Table. 2 The longest sensing distance and pulse width corresponding to different grating space

\begin{tabular}{cccc}
\hline \multicolumn{4}{c}{ width corresponding to different grating space } \\
\hline $\begin{array}{c}\text { Grating } \\
\text { space }(\mathrm{m})\end{array}$ & $\begin{array}{c}\text { Optimal } \\
\text { reflectivity }(\mathrm{dB})\end{array}$ & $\begin{array}{c}\text { Pulse } \\
\text { width(ns) }\end{array}$ & $\begin{array}{c}\text { Sensing } \\
\text { distance }(\mathrm{km})\end{array}$ \\
\hline 10 & -42.4 & 50 & 74.51 \\
20 & -40.3 & 10 & 86.52 \\
30 & -38.9 & 10 & 88.17 \\
40 & -38.1 & 10 & 91.68 \\
50 & -37.2 & 10 & 93.05 \\
60 & -36.6 & 50 & 94.32 \\
70 & -36.1 & 10 & 98.14 \\
\hline \hline
\end{tabular}

\section{CONCLUSION}

In conclusion, we carry out a series of simulations to optimize the parameters to increase the sensing distance of double pulse $\Phi$-OTDR assisted with UWFBG array. The influences of the space interval, the reflectivity, the pulse width and the nonlinear effect to the longest sensing distance are considered in the simulation. Finally, we find out the optimal reflectivity and pulse width corresponding to different space interval to achieve the longest sensing distance.

\section{ACKNOWLEDGMENTS}

This work is supported by the National Natural Science Foundation of China under Grant No. 61627816, 61540017, 61405090 and 61307096, and the Fundamental Research Funds for the Central Universities under Grant No. 021314380087.

\section{REFERENCES}

[1] Q. He, T. Zhu, X. Xiao, B. Zhang, D. Diao and X. Bao,"All Fiber Distributed Vibration Sensing Using Modulated Time-Difference Pulses", IEEE Photon. Technol. Lett. 25,1955-1957, (2013).

[2] J. C. Juarez, E. W. Maier, C. K. Nam and H. F. Taylor, "Distributed fiber-optic intrusion sensor system", J. Lightwave Technol,2081-2087, (2005).

[3] F. Peng, N. Duan, Y. Rao and J. Li, "Real-Time Position and Speed Monitoring of Trains Using Phase-Sensitive OTDR", IEEE Photon. Technol. Lett,2055-2057, (2014).

[4] F. Peng, H. Wu, X.-H. Jia, Y.-J. Rao, Z.-N. Wang and Z.-P. Peng, "Ultra-long high-sensitivity phi-OTDR for high spatial resolution intrusion detection of pipelines", Opt. Express 22,13804-13810, (2014)

[5] H. Chenyuan, W. Hongqiao and B. Wei, "A Novel Interrogation System for Large Scale Sensing Network With Identical Ultra-Weak Fiber Bragg Gratings", J. Lightwave Technol. 32,1406-1411, (2014).

[6] X. Li, Q. Sun, D. Liu, R. Liang, J. Zhang, J. Wo, P. P. Shun and D. Liu, "Simultaneous wavelength and frequency encoded microstructure based quasi-distributed temperature sensor", Opt. Express 20,12076-12084, (2012).

[7] X. Wang, Z. Yan, F. Wang, C. Mou, Z. Sun, X. Zhang and L. Zhang, "SNR enhanced distributed vibration fiber sensing system employing polarization-OTDR and ultra-weak FBGs", IEEE Photonics J. 7,680051, (2015).

[8] Y. Wang, J. Gong, B. Dong, D. Y. Wang, T. J. Shillig and A. Wang, "A Large Serial Time-Division Multiplexed Fiber Bragg Grating Sensor Network", J. Lightwave Technol,2751-2756, (2012).

[9] F. Zhu, Y. Zhang, L. Xia, X. Wu and X. Zhang, "Improved $\Phi-O T D R$ Sensing System for High-Precision Dynamic Strain Measurement Based on Ultra-Weak Fiber Bragg Grating Array", J. Lightwave Technol. 33,4775-4780, (2015).

[10] Derickson, "Fiber optic test and measurement",449-454, (2002).

[11] L. D. Lv,"Research on frequency division multiplexing probe based coherent optical time domain reflectometry", master's thesis, 20-21, (2012).

[12] Y. C. Shen, M. P. Song, X. M. Zhang and K. S. Chen, "Analysis and measurement of stimulated Brillouin scattering threshold in single mode fiber", J. Chinese Lasers,498-500, (2005). 